\title{
La prise en compte des actions sismiques pour le pont Vasco Da Gama
}

\author{
A. PECKER \\ P-DG Géodynamique \\ et Structure \\ 157 , rue des Blains \\ 92220 Bagneux
}

Les profils stratigraphiques et les caractéristiques mécaniques des sols rencontrés à l'emplacement du $2^{e}$ pont sur le Tage à Lisbonne ont nécessité le recours à des études spécifíques pour la définition des sollicitations sismiques à prendre en compte dans le dimensionnement de l'ouvrage. Ces études sont basées sur des calculs non linéaires de propagation d'ondes. Elles ont confirmé la forte influence de la nature des sols sur la réponse en surface avec des amplifications importantes à faible fréquence.

\section{Definition of seismic motions for the Vasco Da Gama bridge}

The soil profiles and the mechanical characteristics of the materials encountered at the location of the 2nd Tagus crossing in Lisbon, called for specific studies for the definition of the design seismic motions. These studies are based on non-linear wave propagation analyses. They confirmed the utmost. importance of the soil characteristics on the ground surface motion with large amplifications at low frequency. 


\section{Introduction}

Le pont Vasco de Gama est situé dans une zone de sismicité relativement importante dont l'événement le plus marquant est le séisme historique de Lisbonne en 1755. Ce contexte particulier, allié à l'importance économique de l'ouvrage, impose que l'agression sismique soit dûment prise en compte dans la conception et le dimensionnement de l'ouvrage.

Dans cet article, on présente la méthodologie avant abouti à la définition de la sollicitation sismique au niveau des fondations de l'ouvrage (pont principal et viaducs).

\section{2}

\section{Définition de la sollicitation sismique}

\section{1}

\section{Position du problème}

Le contexte géotechnique particulier de la vallée du Tage (Wastiaux et al., 1999) constitué d'une couche de vase molle d'une trentaine de mètres d'épaisseur surmontant des dépôts alluvionnaires plus consolidés, puis un substratum rocheux, n'est pas sans rappeler le profil géotechnique rencontré dans la ville de Mexico (AFPS, 1985). Lors du séisme de Michoacan Guerero de 1985, les enregistrements recueillis dans la ville de
Mexico ont mis en évidence une forte amplification du mouvement sismique en tête des dépôts meubles par rapport à ceux recueillis sur les affleurements des dépôts consolidés. La figure 1 présente les enregistrements de ce séisme de 1985.

Au centre de la zone du lac (station SCT), en tête des dépôts alluvionnaires décrits ci-dessus, l'accélération maximale atteint $0,18 \mathrm{~g}$ alors qu'à l'extérieur de la ville (station Tacubaya) elle reste limitée à 0,04 g. Les sols à l'emplacement de la station Tacubaya sont constitués de dépôts plus raides qui correspondent à des affleurements des couches consolidées rencontrées en profondeur ( $>30-40 \mathrm{~m}$ ) dans la zone du lac.

Plus frappante est la différence entre les allures temporelles des deux signaux: celui de SCT est d'allure quasiment monochromatique, avec une période de l'ordre de 2 secondes, et possède une durée très longue, plus de soixante secondes. Par opposition, celui de Tacubaya est plus court et plus erratique ; il résulte de la superposition d'un grand nombre d'harmoniques de périodes différentes : c'est un signal à large bande. Notons que le signal enregistré à Tacubaya est plus conforme à ce qui résulterait de l'application de lois d'atténuation statistique sur un sol « normal » pour un séisme de magnitude 8,1 prenant origine à plus de 400 kilomètres. L'anomalie entre les deux signaux serait constituée par l'enregistrement de SCT si la nature géotechnique des couches de fondation n'était pas prise en considération.

En sismologie de l'ingénieur, il est admis que le mouvement sismique généré en un point donné dépend de trois facteurs principaux:

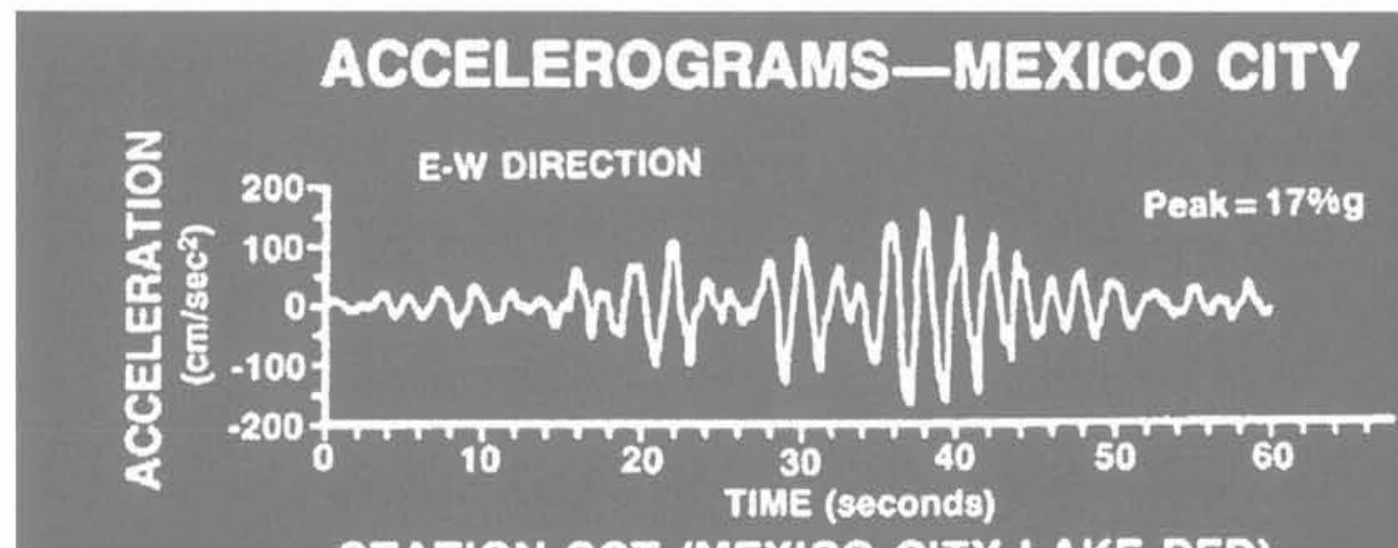

STATION SCT (MEXICO CITY LAKE BED)
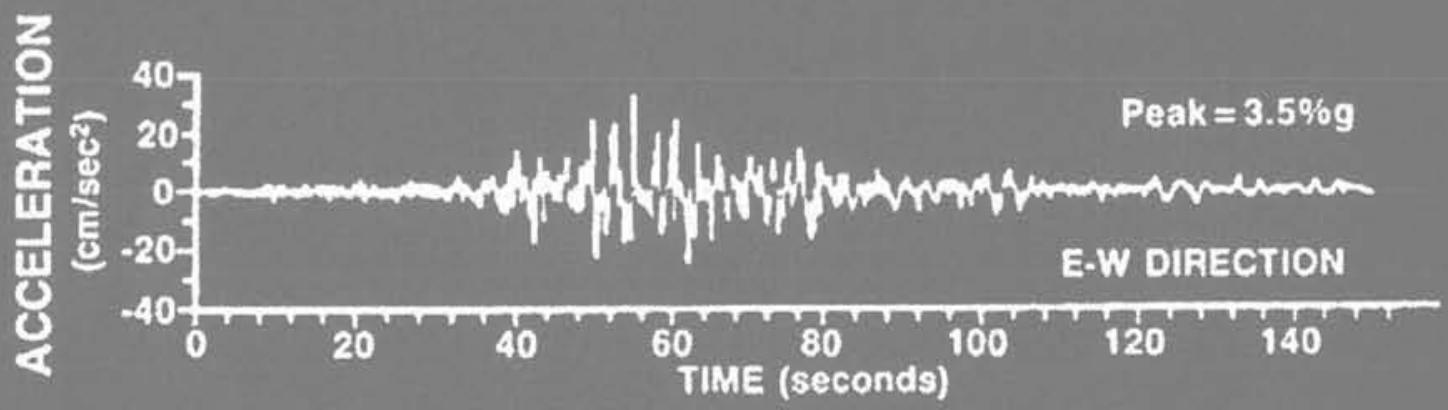

STATION TACUBAYA (MEXICO CITY ROCK SITE)

FIG.1 Enregistrements du séisme de 1985. Ville de Mexico. Seismic records of the 1985 earthquake. Mexico city. 
- un facteur lié à la source du séisme, c'est-à-dire caractérisant l'énergie libérée, le mécanisme de rupture... - un facteur lié au parcours des ondes entre la source (le foyer) et le lieu d'enregistrement (le site) ; idéalement ce facteur reflète toutes les diffractions, polarisations subies par les ondes à tous les interfaces et hétérogénéités rencontrés, atténuation géométrique liée à l'expansion du front d'onde et atténuation anélastique liée au comportement des matériaux traversés :

- un facteur lié aux conditions géologiques et géotechniques à l'aplomb du site.

De toute évidence, pour les enregistrements de la figure 1, compte tenu de la distance (> 400 kilomètres) aux deux sites qui ne sont séparés que de quelques kilomètres, l'impact des deux premiers facteurs est identique ; seul la prise en considération du troisième (conditions locales) permet d'expliquer la différence entre les signaux.

\section{2}

\section{Méthode d'évaluation de la sollicitation sismique}

Compte tenu des éléments présentés ci-dessus, le maitre d'ouvrage avait pris le parti de ne pas définir la sollicitation au niveau de la surface du sol, ce qui aurait requis la connaissance des caractéristiques mécaniques des sols inconnues avec une prévision suffisante au moment de l'appel d'offres, mais en surface d'un affleurement du rocher rencontré sous les dépôts alluvionnaires récents.

Cette donnée était spécifiée sous la forme d'un spectre de réponse d'un oscillateur simple, à $5 \%$ d'amortissement critique. Le spectre est présenté sur la figure 2. Il correspond à un événement 4,5 fois plus important que celui défini par la réglementation portugaise : ce facteur 4,5 avait été fixé pour diminuer, compte tenu de l'importance économique de l'ouvrage, la probabilité de dépassement de la sollicitation sismique. L'accélération maximale au niveau du rocher est de $0,45 \mathrm{~g}$.

Il appartenait au groupement de définir à partir de cette donnée les sollicitations appliquées à l'ouvrage.

Cette définition a comporté les étapes suivantes : - définition d'accélérogrammes synthétiques représentatifs du séisme de référence spécifié au rocher;

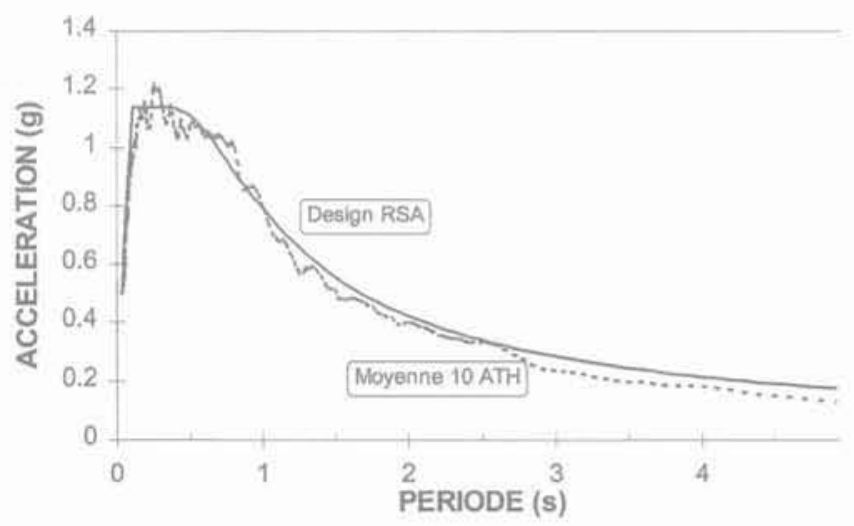

FG. 2 Spectre de réponse au rocher. Bedrok response spectra.
- détermination des profils stratigraphiques, des caractéristiques et du comportement cyclique des différentes couches de sol rencontrées le long de l'ouvrage ;

- calculs linéaires et non linéaires de propagation d'ondes à travers les différents profils pour évaluer le mouvement en surface du sol ;

- études de sensibilité ;

- définition des spectres de dimensionnement en surface.

\section{3}

\section{Accélérogrammes synthétiques}

Dix accélérogrammes ont été générés artificiellement avec pour seul objectif l'obtention d'un spectre aussi proche que possible de celui du séisme de référence. Le spectre moyen de ces dix réalisations est représenté sur la figure 2 et comparé au spectre cible ; les écarts entre spectre moyen et spectre cible n'excèdent pas $10 \%$. Les dix accélérogrammes ainsi générés seront utilisés pour le calcul de propagation d'ondes.

\section{4}

\section{Caractéristiques dynamiques des sols}

Le contenu de la reconnaissance géotechnique ayant permis de définir le profil stratigraphique le long de l'ouvrage et d'évaluer les caractéristiques mécaniques des terrains est décrit par Wastiaux et al. (1999).

On insistera ici plus particulièrement sur les caractéristiques nécessaires aux études dynamiques: module de cisaillement élastique (ou vitesse de propagation des ondes de cisaillement), également appelé module maximal $G_{\max }$; cette donnée caractérise le comportement élastique (à très petite déformation, de l'ordre de $10^{-6}$ ) du matériau. Le sol ayant sous sollicitation sismique un comportement fortement non linéaire, il convenait, par ailleurs, de caractériser ce comportement.

\section{1}

\section{Caractéristiques élastiques}

Elles résultent directement des mesures de vitesses de propagation d'ondes effectuées en place et des essais de colonne résonante réalisés au laboratoire sur échantillons intacts.

Pour les mesures in situ, deux types d'essais ont été utilisés : l'essai cross-hole standard qui mesure, à une profondeur donnée, le temps de parcours des ondes entre deux forages voisins (propagation horizontale) et l'essai au cône sismique qui mesure le temps de parcours des ondes entre la surface et la pointe pénétrométrique lors de l'essai de pénétration.

Les essais de colonne résonante réalisés sous différentes étreintes ont permis de déterminer les lois de variation du module élastique $\left(G_{\text {max }}\right)$ avec l'état de contrainte (Fig. 3). Ces lois ont servi a estimer la valeur de $G_{\max }$ en place à partir du poids volumique des sols en faisant l'hypothèse, confirmée par ailleurs, de sols normalement consolidés.

L'ensemble de ces données a par ailleurs été comparé aux estimations effectuées à partir de corrélations 


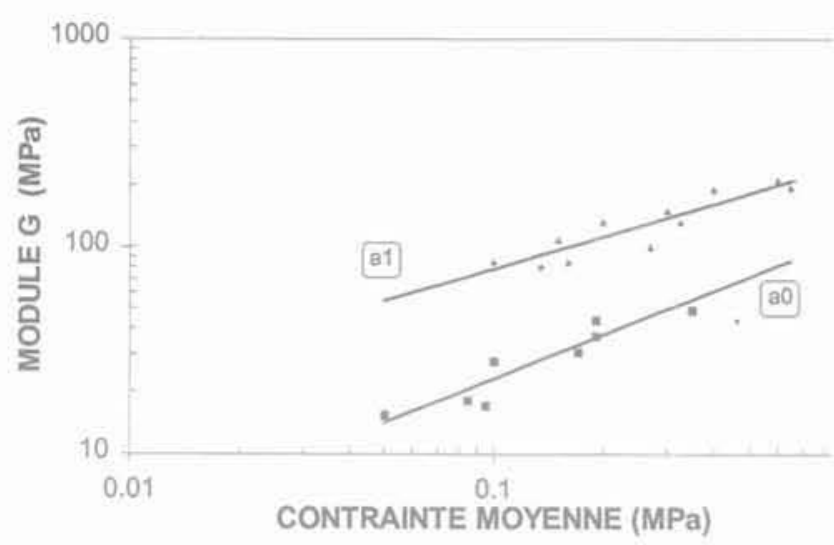

FG. 3 Essais de colonne résonante. Resonant column tests.

expérimentales reliant $\mathrm{G}_{\max }\left(\mathrm{ou} \mathrm{V}_{\mathrm{s}}\right)$ à d'autres caractéristiques mécaniques du matériau.

Pour les matériaux cohérents (couche a) le module $G_{\max }$ a été estimé à partir de la cohésion non drainée $C_{u}$. Les corrélations expérimentales s'écrivent (Weiler, 1988):

$$
\mathrm{G}_{\max }=\mathrm{KC}_{\mathrm{u}}
$$

oủ $\mathrm{K}$ dépend de l'indice de plasticité IP et du degré de surconsolidation OCR de l'argile. Pour le matériau du site :

$$
\mathrm{G}_{\max } \approx(800 \mathrm{à} 1200) \mathrm{C}_{\mathrm{u}}
$$

avec $\mathrm{C}_{0}(\mathrm{kPa})=10+0,23 \sigma^{\prime}(\mathrm{kPa})$

où $\sigma_{v}^{\prime}$ est la contrainte verticale effective (Fig. 4).

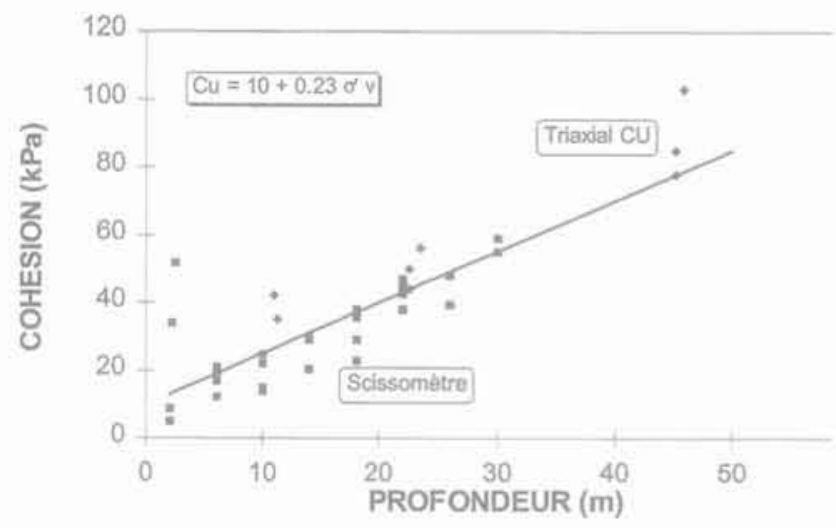

FIG.4 Variation de la cohésion non drainée avec la profondeur.

Undrained shear strength profile.

Pour les matériaux pulvérulents (couche a1, a2b, a3) le module Gmax a été estimé à partir du nombre N de coups SPT (Seed et al., 1984):

$$
\mathrm{G}_{\max }=440 \mathrm{p}_{\mathrm{a}}\left(\mathrm{N}_{1}\right)^{1 / 3}\left(\frac{\sigma_{\mathrm{m}}^{\prime}}{\mathrm{p}_{\mathrm{s}}}\right)^{\mathrm{n}}
$$

où :

$\mathrm{p}_{\mathrm{a}}=$ pression atmosphérique,

$\sigma_{\mathrm{m}}^{\prime}=$ contrainte moyenne effective,

$\mathrm{n}=0,5$.

$N_{1}=N\left(\frac{P_{a}}{\sigma_{v}^{\prime}}\right)^{0,5}$ valeur normalisée de $N$.
L'ensemble de ces informations a permis de définir à l'emplacement de chacune des fondations le profil de variation en fonction de la profondeur de la vitesse de propagation des ondes de cisaillement. Un exemple est donné sur la figure 5 au droit du pylône Sud du pont à haubans. De façon générale, les mesures ou estimations de $V_{\text {s }}$ étaient relativement groupées dans les 30 mètres supérieurs, avec une plus grande dispersion en profondeur. Curieusement, les essais cross-hole fournissaient parfois des valeurs faibles et vraisemblablement erronées; l'exemple de la figure 5 est frappant à cet égard avec une valeur constante de la vitesse sur les 15 premiers métres, en contradiction avec les autres mesures et avec la nature du matériau, homogène et normalement consolidé.

A partir de ces données, deux courbes représentant une estimation haute et une estimation basse des caractéristiques ont été définies ; elles sont représentées en trait plein sur la figure 5 . Ce sont ces courbes qui ont été utilisées dans les études de propagation d'ondes.

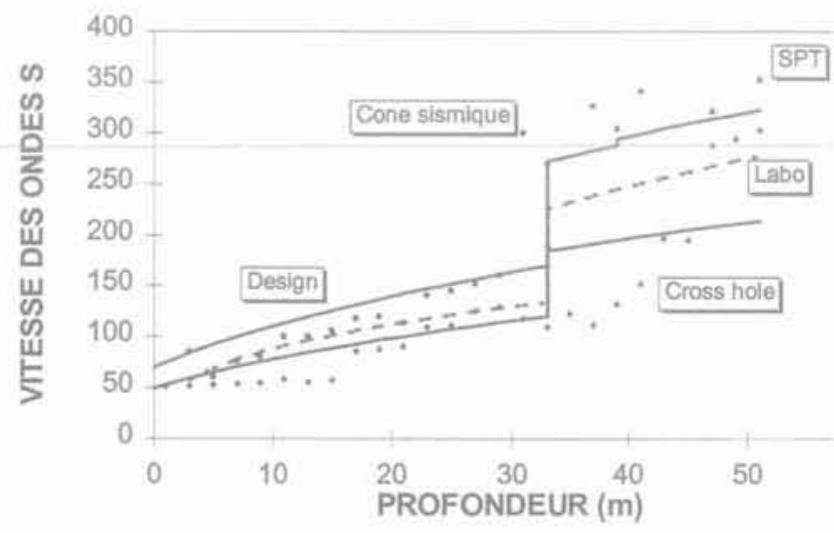

FIG.5 Variation de la vitesse des ondes de císaillement avec la profondeur. Shear wave velocity profile.

\section{2}

\section{Caractéristiques non linéaires}

Elles ont été déterminées essentiellement en vue d'une représentation du comportement non linéaire du sol à l'aide d'une loi de comportement de type viscoélastique linéaire équivalent (Pecker 1984). Dans ce type de modélisation, la courbe effort déformation sous chargement cyclique s'écrit :

$$
G=G_{s}\left[1-2 \beta^{2}+2 i \beta \sqrt{1-\beta^{2}}\right]
$$

où :

$\mathrm{G}_{\mathrm{s}}$ est le module sécant qui dépend de l'amplitude de la déformation de cisaillement $\gamma$; à faible déformation $\mathrm{G}_{\mathrm{s}}=\mathrm{G}_{\max }$, défini au paragraphe 4.1.;

$\beta$ est le pourcentage d'amortissement critique équivalent, également fonction de la déformation.

Les variations de Gs et $\beta$ avec $\gamma$ ont été mesurées dans les essais de laboratoire consistant d'essais de colonne résonante, d'essais triaxiaux cycliques, d'essais de cisaillement simple. Les figures $6 a$ et $6 b$ présentent les mesures obtenues pour le matériau a avec la courbe moyenne retenue pour les études. Les courbes déterminées sur les trois types de matériaux sont données pour les figures $7 \mathrm{a}$ et $7 \mathrm{~b}$. Comme attendu, les matériaux les plus plastiques $\left(\mathrm{a}_{\mathrm{c}}\right.$ ) présentent un com- 

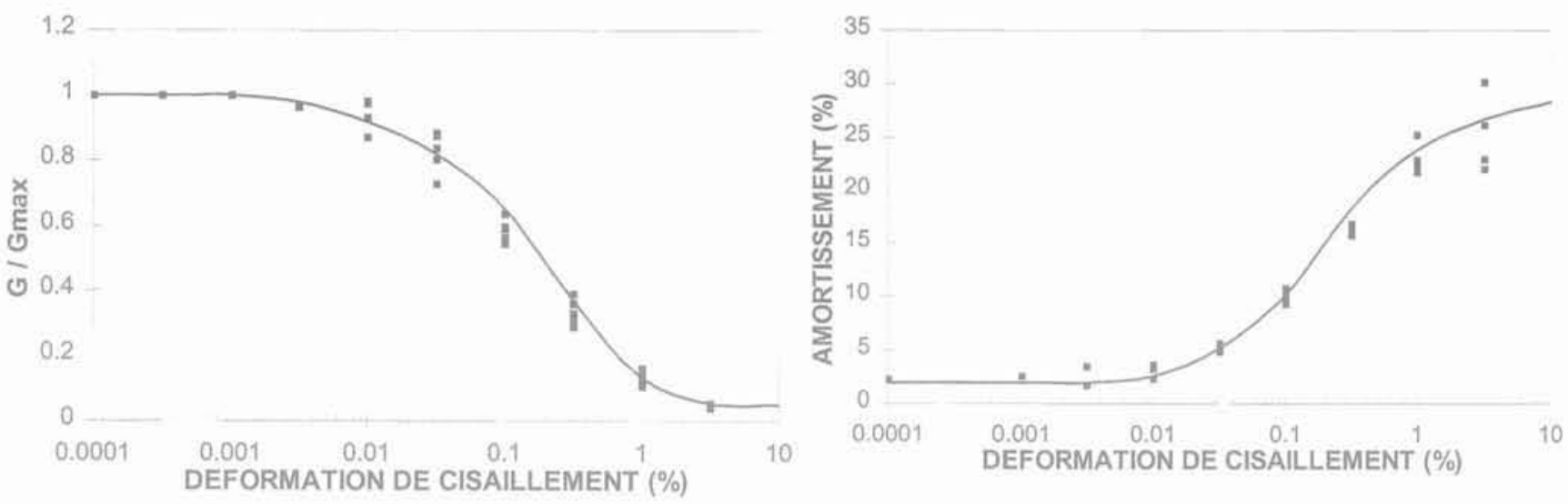

FG. 6 Variation des caractéristiques avec la déformation. Matériau $\mathrm{a}_{0}$. a) module ; b) amortissement. Strain dependence of characteristics. Material $a_{b}$. a) shear modulus ; b) equivalent damping ratio.

portement élastique $\left(\mathrm{G} / \mathrm{G}_{\max }=1,0\right)$ dans un domaine de déformation plus étendu, mais inversement une capacité dissipative, représentée par le paramètre $\beta$, moins élevée. Il a par ailleurs été vérifié que les courbes obtenues sont en bon accord avec celles publiées dans la littérature (Vucetic, Dobry, 1991) pour des matériaux semblables.

Les calculs de propagation d'ondes présentés ciaprès ont également fait appel à une modélisation réellement non linéaire du comportement des sols. Un modèle de comportement type élastoplastique avec écrouissage cinématique et loi d'écoulement non associée a été utilisé. Les paramètres du modèle de Prevost (1985) ont été déterminés à partir des essais de laboratoire mentionnés ci-dessus et ont permis de reproduire fidèlement les courbes de la figure 7 .

\section{5}

\section{Calculs de propagation d'ondes}

\section{1}

\section{Calculs de base}

Ils ont été réalisés en retenant une géométrie unidimensionnelle des couches de fondation. Au droit de chaque appui de l'ouvrage, le profil stratigraphique a été schématisé par une colonne de sol représentant l'empilement vertical de couches horizontales.

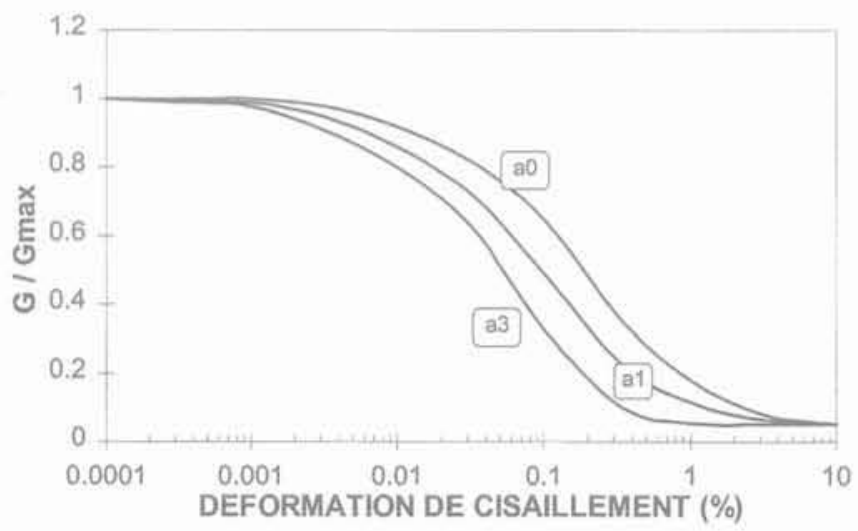

Les lois de comportement utilisées pour la représentation du comportement du sol comprennent: - le modèle linéaire équivalent dans lequel les caractéristiques $G_{\text {s }}$ et $\beta$ sont ajustées itérativement pour être, dans chaque couche, compatibles (au sens de la figure 7) avec la déformation moyenne induite;

- un modèle unidimensionnel non linéaire obtenu par introduction d'un seuil plastique dans la courbe effortdéformation; pour les décharges et recharges successives le modèle obéit aux lois de Masing ; ce modèle est connu sous le nom de Modèle de Iwan (1967);

- un modèle élastoplastique tridimensionnel avec prise en compte du caractère biphasique du sol (modèle de Prevost).

Dans tous les cas, les propriétés élastiques des différents matériaux ont été prises égales soit aux caractéristiques minimales, soit aux caractéristiques maximales (\$ 4.1).

La cinématique du mouvement a été en générale prise unidirectionnelle avec un seul degré de liberté, le déplacement horizontal à chaque nceud. Pour les calculs élastoplastiques, du fait du couplage entre les déformations volumiques (qui dans le cas présent sont faibles du fait de la saturation des matériaux et de leur faible perméabilité) et les déformations de cisaillement, deux degrés de liberté sont assignés à chaque nceud: déplacement horizontal et déplacement vertical.

La sollicitation sismique est définie par les 10 accélérogrammes synthétiques dont les spectres sont voisins de celui du séisme de référence (§ 3$)$.

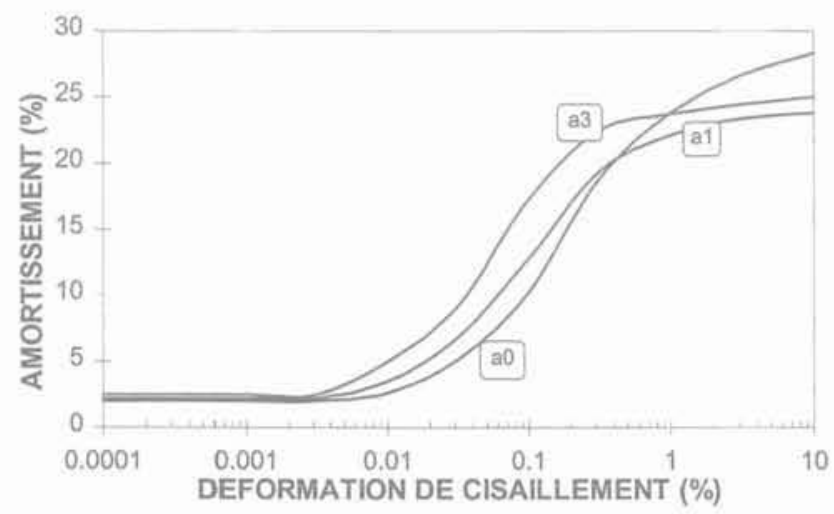

F16.7 Variations des caractéristiques avec la déformation. Tous matériaux. a) module ; b) amortissement, Strain dependence of characteristics. All materials; a) shear modulus ; b) equivalent damping ratio. 


\section{Études paramétriques}

Outre les nombreuses variations déjà prises en compte dans les études de base qui conduisent au droit de chaque appui à 60 calculs (2 jeux de caractéristiques élastiques $\times 3$ modèles de comportement $\times 10$ accélérogrammes), l'influence des paramètres suivants a été étudiée :

- profondeur du substratum rocheux:

- cinématique du mouvement; certains calculs élastoplastiques ont été réalisés en imposant deux sollicitations horizontales, non corrélées, à la colonne de sol. Du fait du couplage déformations volumiques-déformations de cisaillement, la cinématique du mouvement est tridimensionnelle.

\section{6}

\section{Résultats}

La figure 8 représente, à l'emplacement du pont à haubans les spectres de réponses calculés avec le modèle linéaire équivalent pour les profils de sol associés aux caractéristiques élastiques minimales et maximales. Pour un profil stratigraphique donné, le spectre représente la moyenne des spectres calculés avec les 10 accélérogrammes artificiels. On constate qu'à faible période la réponse est contrôlée par les caractéristiques maximales, alors qu'au-delà de trois secondes elle est contrôlée par les caractéristiques minimales. Sur la base de ce modèle de comportement le spectre de calcul à retenir serait constitué par l'enveloppe des deux courbes.

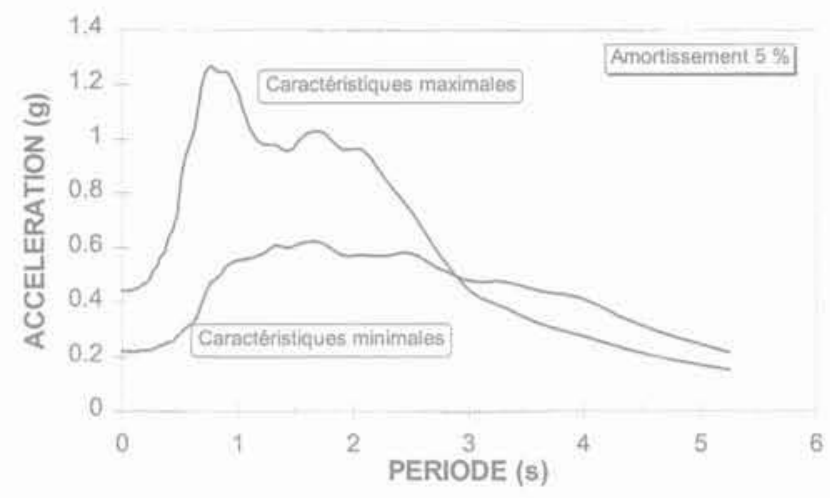

FIG. 8 Spectres de réponse au droit du pont à haubans. Calculs linéaires équivalents. Response spectra at the cable stayed bridge location. Linear equivalent calculations.

La figure 9 met en évidence l'influence de la stratigraphie en comparant les spectres calculés avec le modèle linéaire équivalent le long de l'ouvrage au droit du pont à haubans et en deux emplacements du viaduc central. On note une forte influence de la stratigraphie, plus particulièrement en dessous de 0,5 seconde et entre 1,5 et 2,5 secondes. Pour une période donnée, la réponse maximale dépend de la localisation et ne correspond pas toujours à la même stratigraphie.

La figure 10 compare au droit du pont à haubans la réponse calculée en surface en fonction du modèle de comportement retenu pour les différents matériaux. A

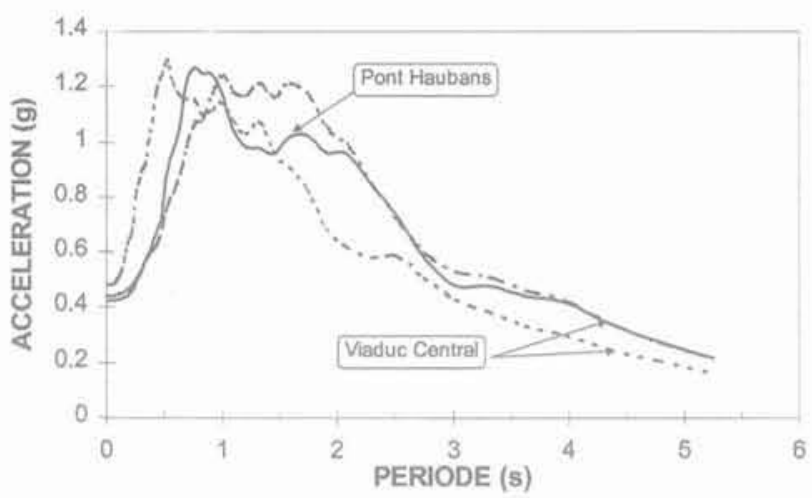

F16.9 Calculs linéaires équivalents. Influence de la stratigraphie.

Linear equivalerit calculations. Influence of stratigraphy.

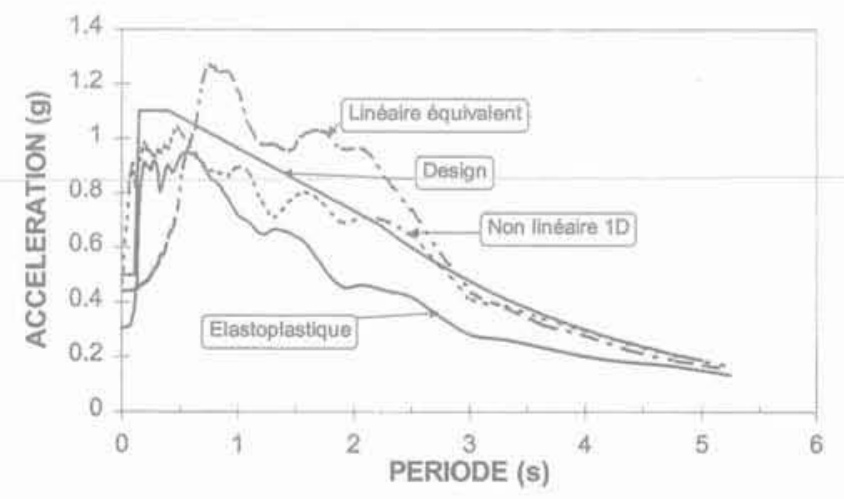

FG. 10 Influence de la loi de comportement du sol. Influence of the soil constitutive model.

faible période $(\mathrm{T}<0,5 \mathrm{~s})$ le modèle linéaire équivalent sous-estime fortement la réponse, alors qu'entre $0,5 \mathrm{~s}$ et $2,5 \mathrm{~s}$ il le surestime par rapport au modèle non linéaire. Cette tendance est connue pour les sollicitations importantes et est inhérente au modèle linéaire équivalent: les hautes fréquences sont filtrées par suite de la prise en compte d'un amortissement équivalent indépendant de la fréquence et calculé en fonction de la déformation moyenne qui est contrôlée par les basses fréquences ; certaines fréquences, correspondant aux fréquences propres de la couche de sol, sont amplifiées.

Les deux modèles non linéaires (1D et élastoplastique) conduisent à des réponses voisines jusqu'à 1,5 seconde et divergent au-delà, le modèle $1 \mathrm{D}$ conduisant à des réponses plus élevées. Cette différence de comportement est due à l'extrapolation qui est faite des courbes effort-déformation à déformation élevée $(>1 \%)$; les essais de laboratoire disponibles ne permettent pas de connaitre cette branche de la courbe. Le modèle $1 \mathrm{D}$ a été calibré en extrapolant « de façon raisonnable » les courbes de la figure 7 alors que le modèle élastoplastique a été calibré sur les données disponibles pour les déformations inférieures à 1\%; le comportement pour les déformations plus élevées résultent alors du modèle choisi. Pour les périodes élevées du spectre qui sont contrôlées par la réponse à déformation élevée du profil de sol, les modèles linéaire et non linéaire 1D donnent des résultats comparables, car ils sont bâtis sur la même extrapolation des courbes de la figure 7 à déformation élevée. 
Devant cette incertitude, il a été décidé de retenir une évaluation raisonnablement conservative pour le spectre de réponse en surface; cette évaluation est donnée sur la figure 10 et enveloppe légèrement le spectre calculé avec le modèle non linéaire 1D.

Les autres études paramétriques réalisées pour le projet ont montré la faible dépendance des spectres calculés sur la profondeur du substratum rocheux ou sur la cinématique choisie. La figure 11 montre, au droit du pont à haubans la différence entre les spectres calculés avec le modèle élastoplastique dans l'hypothèse d'une sollicitation unidirectionnelle ou d'une sollicitation bidirectionnelle. Devant ce faible écart, au regard des autres variations mises en évidence sur les figures 8 à 10 , l'ensemble des études ayant conduit aux spectres de calcul ont été réalisées avec une cinématique unidirectionnelle.

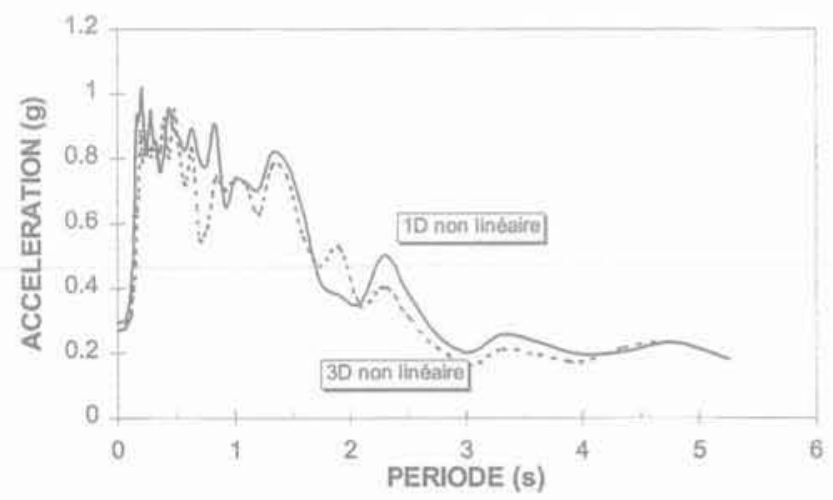

FIG.11 Influence de la cinématique du mouvement sur la réponse.

Influence of the kinematic of motion on the response.

\section{Conclusions}

Les nombreuses études paramétriques réalisées pour l'ouvrage ont conduit à la définition par zones, correspondant à des stratigraphies différentes, de spectres de calcul du mouvement horizontal en surface. Compte tenu des sollicitations sismiques élevées induites par le séisme de référence, il est apparu que les calculs linéaires équivalent ne constituaient pas l'outil approprié pour la détermination de ces spectres ; la détermination des spectres a reposé sur la réalisation de calculs non linéaires.

La figure 12 compare, à l'emplacement du pont à haubans le spectre de réponse horizontal à la surface à celui du mouvement de référence imposé au niveau d'un affleurement du substratum rocheux. L'influence des caractéristiques mécaniques des sols est apparente avec une forte amplification de la réponse pour les périodes supérieures à la seconde; en haute fréquence, la réponse est par contre légèrement filtrée par rapport à celle au rocher. Cette amplification justifie, a posteriori, la nécessité des études présentées ci-dessus pour la définition du spectre de calcul.

Pour le mouvement vertical, aucun calcul n'a été réalisé pour la définition du spectre en surface. En effet, le mouvement vertical résulte principalement de la propagation verticale d'ondes de compression; le matériau étant saturé, il est pratiquement incompressible et le mouvement se transmet depuis le rocher sans être altéré par la propagation. Le spectre vertical sur la figure 12 correspond donc à celui de la sollicitation verticale au niveau du rocher.

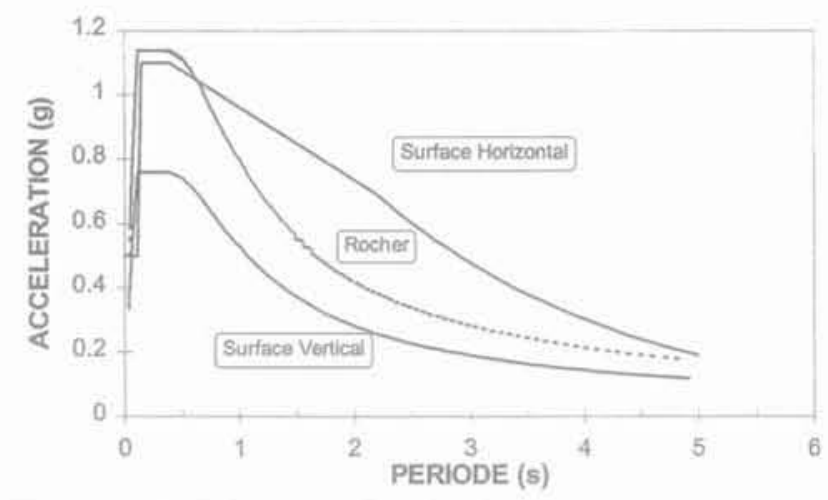

FG. 12 Spectres de calcul. Design spectra.

\section{$\overline{B i b l i o g r a p h i e}$}

Association Française du Génie Parasismique. - «le séisme du Mexique du 19 septembre 1985 m. Compte rendu de mission. 1985.

Iwan W.D. - $\alpha$ On a class of models for the yielding behavior of continuous and composite systems 2. Journal of Applied Mechanics, ASME, vol. 34 n" E3, 1967.

Pecker A. - Dynamique des sols. Presses de I'ENPC 1984

Prevost J.H. - "A simple plasticity theory for frictional cohesionless soils m. Soil Dynamics and Earthquake Engineering. vol. 4, n² 1, 1985, p. 9-17.

Seed H.B. Wong R.T. Idiriss IM. Tokimatsu K. - - Moduli and damping factors for dynamics analyses of cohesionless soils ग. Earthquake Engineering Research Center, Report EERC 84-14, 1984.

Vucetic M., Dobry R. - i Effect of soil plasticity on cyclic response $\mathrm{x}$. Journal of
Geotechnical Engineering, ASCE. vol. 117, n*1, 1991

Wastiaux M., Ducroq J., Corbetta F, - « Les pleux maritimes du pont Vasco da Gama n. Revue francaise de gếotechnique, $\mathrm{n}^{\circ} 87,1999$

Weller W.A Jr. - « Small strain shear modulus of clay $x$. Fecent advances in ground motion evaluation. ASCE Geotechnical Publication $n^{2} 20,1988$, p. 331 345 . 